\title{
Hashimoto Thyroiditis Coexistent with Papillary Thyroid Carcinoma
}

CAlOGERO CIPOLla, M.D., Ph.D., LUIGI SANDONATO, M.D., GIUSEPPA GRACEFFA, M.D., SALVATORE FRICANO, M.D., ADRIANA TORCIVIA, M.D., SALVATORE VIENI, M.D., Ph.D., STEFANIA LATTERI, MARIO ADELFIO LATTERI, M.D.

From the University of Palermo, Department of Oncology, Division of General and Oncological Surgery Policlinico "P. Giaccone" Palermo, Italy

Several studies report a higher rate of papillary thyroid carcinomas (PTC) in patients with Hashimoto thyroiditis (HT), indicating a possible correlation between the two diseases. We studied a group of 89 subjects undergoing surgery for thyroid carcinomas compared with a control group of 89 subjects operated on for normofunctioning goiter, and a second group of 47 patients undergoing total thyroidectomy for HT. Association with HT was found in 19 of the 71 PTC subjects $(26.7 \%)$ and in 8 goiter patients $(8.9 \%)$, which was a significant difference $(P<0.02)$. Thirteen of the HT patients, mostly with the nodular form, showed coexistent PTC (27.6\%). HT and PTC coexisted in several morphological, immunohistochemical, and biomolecular aspects; increased incidence of PTC in HT patients might therefore indicate that HT is a precursor of thyroid cancer. Further studies are required, however, in order to confirm this hypothesis; until then, HT patients should undergo careful clinical and technical follow-up.

$\mathrm{H}$ ASHIMOTO THYROIDITIS (HT), described for the first time in $1912{ }^{1}$ is an autoimmune disease, affecting 5 per cent of the general population; it involves widespread lymphocyte infiltration, fibrosis, and parenchymal atrophy. It is about 15 times more common in women than in men and is generally diagnosed in subjects from 30 to 50 years of age. More than 90 per cent of such cases show high antiperoxidase and antithyroglobulin antibody titers. ${ }^{2}$

HT occurs in two different clinical forms: nodular or diffuse. Treatment is almost always medical, especially in patients with hypo- or hyperthyroidism. Surgery is indicated in patients when an increase in glandular volume causes compressive symptoms, when drug therapy has proved to be unsatisfactory, and, above all, when there is suspected neoplastic degeneration of one or more nodules.

The association between HT and PTC was first described by Dailey et al. in $1955 .^{3}$ Since then, numerous authors $^{4-7}$ have reported a coexistence between the two diseases. Nevertheless, although these two diseases of the thyroid have a great many morphological, immunohistochemical, and, even more important, biomolecular features in common, the pathological significance and possible clinical implications of

Address correspondence and reprint requests to Dr. Calogero Cipolla, M.D., Dipartimento di Oncologia, Divisione di Chirurgia Generale ed Oncologica, A.O.U. Policlinico "Paolo Giaccone," Via del Vespro 129, 90127 Palermo, Italy. the correlation between them are still not fully understood.

\section{Patients and Methods}

The aim of our study was to evaluate the correlation between HT and PTC. We performed a retrospective analysis of two groups; the first was made up of 89 patients who had undergone total thyroidectomy for carcinomas of the thyroid and the second of 47 subjects who had undergone total thyroidectomy after a preoperative diagnosis of HT. These two groups were part of a wider series of 1,012 patients who underwent thyroidectomy in our division from January 1993 to December 2003. In all cases, the preoperative diagnostic workup included a thyroid hormone assay, ultrasound and scintigraphic examinations of the thyroid, and a cytological examination of the gland by means of echo-guided FNA; the clinical diagnosis of HT was based on the increase of serum levels of antiperoxidase, antimicrosome and antithyroglobulin antibodies.

\section{Group 1}

Of the 89 patients who had undergone surgery for carcinomas of the thyroid, 68 were women and 21 were men, with a mean age of 47 years (range, 21 to 77). Seventy-one $(79,8 \%)$ of these were papillary carcinomas (PTC), 12 of which were occult, seven (7, $8 \%)$ follicular carcinomas, three $(3,3 \%)$ anaplastic carcinomas, four $(4,5 \%)$ medullary carcinomas, two 
TABLE 1. Hashimoto's Thyroiditis Coexistent with PTC (Group 1)

\begin{tabular}{cccc}
\hline & PTC & Goiter & $P$ \\
\hline Coexistent & $26.7 \%$ & $8.9 \%$ & $<0.02$ \\
& (19/71 patients) & (8/89 patients) & \\
\hline
\end{tabular}

PTC, papillary thyroid carcinoma.

$(2,2 \%)$ insular carcinomas, and two $(2,2 \%)$ oncocyte carcinomas. This group of patients was compared with a similar group of 89 patients of the same sex and age who had undergone surgery for normofunctioning nodular goiter during the same period.

\section{Group 2}

Of the 47 patients undergoing radical thyroidectomy after a preoperative diagnosis of HT, 44 were women and 3 were men, with a mean age of 44.7 years (range, 20 to 65$)$. Nineteen cases $(40.4 \%)$ were nodular HT, $23(48.9 \%)$ were diffuse, and $5(10.6 \%)$ were in patients with normofunctioning goiter who had already been followed up for some time. Of the 19 patients with nodular HT, surgical treatment was chosen in 8 because the FNA results produced a cytological indication of carcinomas, in 10 patients because one or more nodules had increased in volume, and in 1 patient because of the appearance of compressive symptoms. Of the 23 patients with diffuse HT and the 5 with HT developing on a preexistent goiter, 25 underwent surgery because of compressive symptoms and 3 patients with an associated hyperthyroidism underwent surgery, because of insufficient response to drug therapy.

In all these patients, total thyroidectomy was performed. The histopathological diagnosis of HT was based on the presence of diffuse, chronic, inflammatory infiltrate containing lymphocytes, germinative plasma cells and centers, fibrosis, and thyroid cells enlarged with eosinophilic cytoplasm and a large number of mitochondria.

\section{Results}

\section{Group 1}

Of the 89 patients operated on for thyroid carcinomas, histological examination of the gland showed the concomitant presence of a histopathological picture suggesting HT. This was observed only in the 71 patients with PTC, with an incidence rate of association between HT and PTC of 26.7 per cent. In one patient $(1.4 \%)$, the PTC was associated with Basedow disease and in one $(1.4 \%)$ with multinodular hyperfunctioning goiter; in the remaining 50 patients, the PTC either presented as a single nodule or else was associated with a histopathological picture indicating goiter (70.4\%).

In the control group, made up of 89 patients operated on in the same period for normofunctioning nodular goiter, only in 8 cases $(8.9 \%)$ did we observe the concomitant presence of histopathological pictures suggesting HT.

The association between HT and PTC, therefore, was significantly higher in the group of patients who had undergone surgery for thyroid carcinomas than in those operated on for goiter $(P<0.02)$ (Table 1$)$.

\section{Group 2}

Of the 47 patients who underwent total thyroidectomy after a diagnosis of HT, histological examination produced a diagnosis of differentiated carcinomas of the thyroid in 13 patients $(27.6 \%)$, which in all cases proved to be PTC, with a mean diameter of $9.6 \mathrm{~mm}$ (range, 1 to $25 \mathrm{~mm}$ ); in no case was a laterocervical lymphoadenopathy present. PTC was found in 11 of the 19 patients $(57.9 \%)$ affected by nodular HT (4 involving an occult tumor) and $2(8.7 \%)$ of the 23 patients with diffuse HT (in both these patients the carcinoma was occult). No case of PTC was found in the group of 5 patients in whom the HT had developed on a preexistent nodular goiter.

In the remaining 34 patients operated on for HT, 2 cases $(6.1 \%)$ showed an association between microfollicular adenoma while 5 cases $(15.2 \%)$ showed coexistent histopathological results indicating goiter.

It is interesting to note that 11 of the 13 cases of PTC $(84.6 \%)$ were found in patients with nodular HT, and only $2(15.4 \%)$ in the patients with diffuse HT $(P<0.001)$, indicating a significantly higher risk of the development of PTC on the nodular variant of HT. The sex and age of the patients nor the time lapse

TABLE 2. Hashimoto's Thyroiditis Coexistent with PTC (Group 2)

\begin{tabular}{lccc}
\hline & HT Nodular Variant & HT Diffuse Variant & $P$ \\
\hline \multicolumn{1}{c}{ Coexistent PTC } & $84.6(11 / 13$ PTC) & $15.4 \%(2 / 13$ PTC) & $<0.001$ \\
& Coexistent HT and PTC & HT & $P$ \\
\hline Mean age & 45.7 & 46.1 & NS \\
M/F ratio & $1 / 12$ & $2 / 22$ & \\
Time lapse between HT diagnosis and surgery & 22.9 mesi (range, $0-96)$ & 32.1 mesi (range, $0-123)$ & \\
\hline
\end{tabular}

HT, Hashimoto thyroiditis; PTC, papillary thyroid carcinoma; NS, not significant. 
occurring between the diagnosis of HT and surgery seemed to be at all significant. In fact, in the group of 13 patients with an association between HT and PTC, the male/female ratio was 1:12 and the mean age was 45 years, while in the 34 patients in whom HT was not associated with PTC, the male/female ratio was 2:22 and the mean age was 44.5 years. In the patients operated on for HT associated with PTC, the HT diagnosis had been made on average 22.9 months before surgery (range, 0 to 96 months), whereas in the patients with HT not associated with PTC, the diagnosis of HT had been made on average 32.1 months before surgery (range, 0 to 123 months).

None of the 11 patients with PTC proved to have a laterocervical lymphoadenopathy. The mean followup of patients diagnosed with PTC was 56.7 months (range, 6 to 102 months); during this period no neoplastic relapse was observed (Table 2).

\section{Discussion}

An association between HT and differentiated thyroid carcinomas has been reported by several authors from the 1950s up to the present time, although with various incidence rates, probably due not only to the different genetic and environmental factors involved, but above all to the various anatomopathological definitions of HT used in the different studies (Table 3).

The aim of our own study was to verify the association between HT and PTC by evaluating two series of patients operated on in our institute from 1993 to 2003. In the first series, we compared the different rate of histopathological pictures indicating HT found in patients operated on for thyroid carcinomas and in a control group made up of patients who had undergone

TABLE 3. Hashimoto's Thyroiditis Coexistent with Thyroid Carcinoma

\begin{tabular}{|c|c|}
\hline Authors & $\begin{array}{c}\text { Coexistent HT } \\
\text { and PTC }(\%)\end{array}$ \\
\hline Dailey et al. $1955^{3}$ & 17.7 \\
\hline Pollock and Sprong $1958^{6}$ & 9.6 \\
\hline Meier et al. $1959^{8}$ & 1 \\
\hline Woolner et al. $1959^{7}$ & 3 \\
\hline Schlicke et al. $1960^{9}$ & 8.7 \\
\hline Shands $1960^{10}$ & 7 \\
\hline Chesky et al. $1962^{11}$ & 11.1 \\
\hline Crile and Hazard $1962^{12}$ & 0.5 \\
\hline Hirabayashi and Lindsay $1965^{4}$ & 22.5 \\
\hline Holmes et al. $1977^{5}$ & $\begin{array}{r}22.5 \\
8.3\end{array}$ \\
\hline Figg et al. $1978^{13}$ & 19 \\
\hline Ott et al. $1985^{14}$ & 32 \\
\hline Sclafani et al. $1993^{15}$ & 17 \\
\hline Loh et al. $1999^{16}$ & 20.3 \\
\hline Singh et al. $1999^{2}$ & 15 \\
\hline Kebebew et al. $2001^{17}$ & 30 \\
\hline
\end{tabular}

HT, Hashimoto thyroiditis; PTC, papillary thyroid carcinoma. surgery for normofunctioning goiter. In the second series of patients, we evaluated the incidence rate of PTC found in patients operated on after a preoperative diagnosis of HT.

Association with HT was observed in 19 of 71 patients $(26.7 \%)$ who underwent surgery for PTC, and in only 8 of 89 patients $(8.9 \%)$ operated on for thyroidectomy for normofunctioning nodular goiter; in agreement with the results reported by several other authors, ${ }^{18-21}$ this confirmed the suggestion that there was a significantly higher association with HT in patients operated on for PTC than in those undergoing surgery for goiter $(P<0.02)$.

A meta-analysis conducted by Singh et al. ${ }^{2}$ demonstrated that the incidence rate of HT is 2.77 times higher in PTC patients than in subjects affected by benign thyroid diseases. Furthermore, in patients with thyroid carcinomas, the incidence rate of association with HT is 1.99 times higher in those with PTC than in subjects with other histopathological forms of thyroid carcinomas, although indirectly, these results suggest that patients with HT might well present a stronger predisposition towards the development of PTC.

This hypothesis is reinforced by the fact that a large number of biomolecular studies have shown the presence of mutation products of the oncogens RET/PTC — a specific genetic alteration for PTC-in patients with HT but without clinically manifest tumors. ${ }^{22,23}$

Several studies involving the expression of $\mathrm{p} 63$ proteins, which generally intervene in the differentiation processes of the stem cells and which are not present in normal thyroid tissue, have shown a higher expression of such proteins in a great many cases both of HT and of PTC, which is a further indication that there is most probably a link between the two diseases. ${ }^{24}$

Moreover, several other studies have shown the presence of both lymphocyte infiltrate and of classical HT in association with 30 per cent to 58 per cent of PTC cases and only in 20 per cent of follicular carcinomas and in 14 per cent of follicular adenomas. ${ }^{17,25,26}$ These data are a further confirmation of the hypothesis that PTC pathogenesis involves an autoimmunity mechanism stimulated by lymphocyte infiltration of the thyroid gland tissue. Furthermore, several studies regarding the effects of certain factors such as race and sex on the presence of lymphocyte infiltrate in PTC, indicative of autoimmune thyroiditis, have shown that this is significantly higher in Japanese patients of both sexes and in both white and black American women with PTC than in the same populations affected by multinodular goiter and follicular adenomas. ${ }^{27}$ This result is in agreement with several epidemiological data that report a higher rate, not only of 
HT, but also a predominance of PTC associated with HT, in women than in men. ${ }^{9}, 11,14,16,18$

With regard to their clinical course, thyroid tumors associated with HT do not seem to be any more aggressive than nonassociated forms. In the series studied by Eisenberg et al., ${ }^{19}$ none of the patients diagnosed with an association between a thyroid carcinoma and HT developed either disease relapse or metastases during the mean follow-up period of 74 months. In our own cases, with a mean follow-up of 56.7 months (range, 6 to 102 months), there was no disease relapse in any of the 19 patients with concomitant HT and PTC or in the 13 subjects operated on for HT when papillary carcinomas were was found.

Ott et al. ${ }^{14}$ reported that carcinomas of the thyroid were found in 47 (32\%) of 146 patients who had undergone surgery for HT associated with a solitary cold nodule of the thyroid gland. McKee et al. ${ }^{28}$ performed histological analysis in 27 of 115 patients with a diagnosis of HT and found 12 cases of concomitant thyroid cancers (10\%), made up of four papillary carcinomas, one follicular carcinoma, and 7 thyroid lymphomas.

In our study, we found an association between HT and thyroid carcinomas, all PTCs, in 13 of 46 patients $(28.2 \%)$ who had undergone thyroidectomy with a preoperative diagnosis of HT. We did not observe any significant differences with regard to age, sex, and the time lapse from the diagnosis of HT until the moment of surgery between these 13 patients and the remaining 33 subjects. An important correlation, however, seemed to exist between the nodular variant of HT and PTC. We found, in fact, that 11 of the 13 papillary carcinomas $(84.6 \%)$ were in patients with this form of HT, while only in 2 patients $(15.4 \%)$ was this type of carcinoma present in patients with diffuse HT. Analysis of this result indicates a much higher risk of the development of PTC in subjects with nodular HT $(P<0.001)$.

In conclusion, therefore, the increased incidence reported in literature of thyroid carcinomas on the nodular form of HT suggests that HT might well be considered as a precursor of the disease. ${ }^{15,28,29}$ The majority of such tumors are PTCs, although an association with follicular, indifferentiated, or medullary carcinomas has also frequently been reported. ${ }^{4,11,15}$ Furthermore, PTC and HT have several morphological, immunohistochemical, and, even more important, biomolecular features in common. Although HT is a benign disease, it may represent the site of RET/PTC rearrangements, an early, but extremely specific marker associated with PTC.

On the basis of these considerations, therefore, should HT be deemed a submicroscopic homologue of PTC? Is there any possibility of establishing a valid criterion for the selection of those patients with HT at higher risk of developing thyroid carcinomas? So far, there are no answers to these questions; there is no doubt that further studies and research are necessary in order to establish the predictive value of RET/PTC rearrangement and of several other markers coexisting in patients with HT, PTC, and other forms of thyroid cancers. Since, however, the annual incidence of carcinomas of the thyroid is 1 male and 2.6 females per 100,000 subjects, extremely long trials involving an enormous number of patients ${ }^{29}$ would therefore be involved.

At the present time, the only possible approach consists in careful, accurate follow-up of HT patients, and especially those with the nodular variant of the disease, not only from the clinical point of view, but above all, with cytological examination of the nodules. The surgical approach should always implicate total thyroidectomy, since it has been reported ${ }^{20}$ that cases of PTC associated with HT are much more frequently multicentric $(93 \%)$ than the nonassociated form of PTC $(50 \%)$.

\section{REFERENCES}

1. Hashimoto H. Zur Kenntiss der lymphomatosen veranderung der Schilddrusse struma lymphomatosa. Arch Klein Chir 1912; 97:218-48. (Translated in Levit T. The Thyroid: A Physiological, Clinical and Surgical Study. London: E. \& S. Livingston, 1954, pp 547-61.)

2. Singh B, Shaha AR, Trivedi H, et al. Coexistent Hashimoto's thyroiditis with papillary thyroid carcinoma: impact on presentation, management, and outcome. Surgery 1999;126:1070-7.

3. Dailey ME, Lindsay S, Skahen R. Relation of the thyroid neoplasm to Hashimoto's disease of the thyroid gland. Arch Surg 1955;70:291-7.

4. Hirabayashi RN, Lindsay S. The relation of thyroid carcinoma and chronic thyroiditis. Surg Gynecol Obstet 1965;121: 243-52.

5. Holmes HS, Kreutes A, O'Brien PH. Hashimoto's thyroiditis and its relationship to other thyroid diseases. Surg Gynecol Obstet 1977;144:887-90.

6. Pollock WR, Sprong DH Jr. The rationale of thyroidectomy for Hashimoto's thyroiditis: a premalignant lesion. West J Surg 1958;66:17-20.

7. Woolner LB, McConaley WM, Beahrs OH. Struma lymphomatosa (Hashimoto's thyroiditis) and related thyroidal disorders. J Clin Endocrinol Metab 1959;19:53-8.

8. Meier DW, Woolner LB, Beahrs OH, McConahey WM. Parenchymal findings in thyroidal carcinoma: pathologic study of 256 cases. J Clin Endocrinol 1959;19:162-71.

9. Schlicke CP, Hill JE, Schultz GF. Carcinoma in chronic thyroiditis. Surg Ginecol Obstet 1960;111:552-6.

10. Shands WC. Carcinoma of the thyroid in association with struma lymphomatosa. Ann Surg 1960;151:675-82.

11. Chesky VE, Hellwing CA, Welch JW. Cancer of the thyroid associated with Hashimoto's disease: an analysis of forty-eight cases. Am Surg 1962;28:678-85. 
12. Crile G Jr., Hazard JB. Incidence of cancer in struma lymphomatosa. Surg Gynecol Obstet 1962;115:101-3.

13. Figg DM, Bratt HJ, van Vliet PD, Dean RE. Thyroid cancer: diagnosis and management based on a review of 142 cases. Am J Surg 1978;135:671-4.

14. Ott RA, Calandra DB, McCall A, et al. The incidence of thyroid carcinoma in patients with Hashimoto's thyroiditis and solitary cold nodules. Surgery 1985;98:1202-6.

15. Sclafani AP, Valdes M, Cho H. Hashimoto's thyroiditis and carcinoma of the thyroid: optimal management. Laryngoscope 1993;103:845-9.

16. Loh KC, Greenspan FS, Dong F, et al. Influence of lymphocytic thyroiditis on the prognostic outcome of patients with papillary thyroid carcinoma. J Clin Endocrinol Metab 1999;84: 458-63.

17. Kebebew E, Treseler PA, Ituarte PHG, Clark OH. Coexisting chronic lymphocytic thyroiditis and papillary thyroid cancer revisited. World J Surg 2001;25:632-7.

18. Ott RA, McCall AR, McHenry C, et al. The incidence of thyroid carcinoma in Hashimoto's thyroiditis. Am Surg 1987;53: 442-5.

19. Eisenberg BL, Hensley SD. Thyroid cancer with coexistent Hashimoto's thyroiditis. Arch Surg 1989;124:1045-7.

20. Asanuma K, Sugenoya A, Kasuga Y, et al. The relationship between multiple intrathyroidal involvement in papillary thyroid carcinoma and chronic non-specific thyroiditis. Cancer Lett 1998; 122:177-80.
21. MacLachlan SM. Rapaport B Genetic and epitopic analysis of thyroid peroxidase (TPO) autoantibodies: markers of the human thyroid autoimmune response. Clin Exp Immunol 1995;101: 200-6.

22. Arif S, Blanes A, Diaz-Cano SJ. Hashimoto's thyroiditis share features with early papillary thyroid carcinoma. Histopathology 2002;41:357-62.

23. Wirtshafter A, Schmidt R, Rosen D, et al. Epression of the RET/PTC fusion gene as a marker of papillary carcinoma in Hashimoto's thyroiditis. Laryngoscope 1997;107:95-100.

24. Unger P, Ewart M, Beverly YW, et al. Expression of p63 in papillary thyroid carcinoma and in Hashimoto's thyroiditis: a pathobiologic link? Hum Pathol 2003;34:764-9.

25. Tamimi DM. The association between chronic lymphocytic thyroiditis and thyroid tumors. Int J Surg Pathol 2002;10:141-6.

26. Schäffer A, Palitzsch KD, Seiffarth $C$, et al. Coexistent thyroiditis is associated with lower tumours stage in thyroid carcinoma. Eur J Clin Invest 1998;28:838-44.

27. Okayasu I, Fujiwara M, Hara Y, et al. Association of chronic lymphocytic thyroiditis and thyroid papillary carcinoma. A study of surgical cases among Japanese and white and African Americans. Cancer 1995;76:2312-8.

28. McKee RF, Krukowski ZH, Matheson NA. Thyroid neoplasia coexistent with chronic lymphocytic thyroiditis. Br J Surg 1993;80:1303-4.

29. Parkin D, Pisani P, Falrlay J. Global cancer statistic. CA Cancer J Clin 1999;49:33-64. 